\title{
Possible geometries of afterglow generation in the gamma-ray burst GRB 990705
}

\author{
X. Y. Wang, Z. G. Dai, and T. Lu
}

\author{
Department of Astronomy, Nanjing University, Nanjing 210093, PR China \\ e-mail: daizigao@public1.ptt.js.cn; tlu@nju.edu.cn
}

Received 23 July 2002 / Accepted 9 January 2003

\begin{abstract}
The absorption feature detected in the prompt X-ray emission of GRB 990705 has important consequences for its circum-burst environment and therefore on its afterglow. Here we investigate whether the circum-burst environment constrained by the absorption feature could be consistent with the observed $H$-band afterglow, which exhibits an earlier power law decay $\left(F \propto t^{-1.68}\right)$ but a much faster decay $\left(\alpha>2.6 ; F \propto t^{-\alpha}\right)$ about one day after the burst. Two possible geometries of the afterglowemitting regions are suggested: 1) afterglow emission produced by the impact of the fireball on the surrounding torus, which serves as the absorbing material of the X-ray feature, as would be expected in the models involving that a supernova explosion precedes the gamma-ray burst by some time; 2) afterglow emission produced in the dense circum-burst medium inside the torus. In case 1), the faster decay at the later time is attributed to the disappearance of the shock due to the counter-pressure in the hot torus illuminated by the burst and afterglow photons. For case 2), the circum-burst medium density is found to be very high $\left(n \gtrsim 10^{4}-10^{5} \mathrm{~cm}^{-3}\right)$ if the emitting plasma is a jet or even higher if it is spherical. Future better observations of afterglows of GRBs that have absorption features might make it possible to make a more definite choice between these two scenarios.
\end{abstract}

Key words. gamma rays: bursts - line: formation - radiation mechanism: nonthermal

\section{Introduction}

There is increasing observational evidence favoring the existence of absorption and emission lines in the X-ray spectra of gamma-ray bursts (GRBs) and their afterglows. Emission or absorption features can provide a fundamental tool for studying the close environment of GRBs (e.g. Mészáros \& Rees 1998; Lazzati et al. 1999, 2002; Böttcher \& Fryer 2001). To date, five bursts have shown evidence for iron or lighter element emission lines during the X-ray afterglow (GRB 970508, Piro et al. 1998; GRB 970828, Yoshida et al. 1999; GRB 991216, Piro et al. 2000; GRB000214, Antonelli et al. 2000; GRB011211, Reeves et al. 2002) and one (GRB 990705; Amati et al. 2000; hereafter A2000) displays a transient absorption feature at $3.8 \mathrm{KeV}$ during the burst itself.

A few models for emission lines in the X-ray afterglows have been suggested (see Piro 2002 for a review), including the "distant reprocessor scenario" and "nearby reprocessor scenario". In the former, the line-emitting gas is located at $R \gtrsim 10^{15} \mathrm{~cm}$ with the line variability time corresponding to the light travel time between GRB and the reprocessor (Lazzati et al. 1999; Piro 2000; Weth et al. 2000). This scenario needs the presence of an iron-rich dense medium with iron mass $M_{\mathrm{Fe}} \gtrsim 0.01 M_{\odot}$. The most straightforward picture is the one in which an SN-like explosion occurs some time before the formation of the GRB. The GRB may be produced

Send offprint requests to: X. Y. Wang, e-mail: xywang@nju.edu.cn by the collapsing of the rotationally-supported newborn massive neutron star into a black hole (Vietri \& Stella 1998), or the phase transition to a strange star (Wang et al. 2000a). In the latter scenario, the line emission is attributed to the interaction of a long-lasting relativistic outflow from the central engine with the massive star progenitor stellar envelope at distances $R \lesssim 10^{13} \mathrm{~cm}$ (Mészáros \& Rees 2000; Rees \& Mészáros 2000).

While different scenarios have been suggested to explain the emission line, the properties of the transient absorption feature, as in GRB 990705, strongly point to a unique circum-burst environment (Lazzati et al. 2001; Böttcher et al. 2002), i.e., 1) iron-rich absorbing matter of a few solar masses (such as the young supernova remnant shell) lies between $10^{16}$ and $10^{18} \mathrm{~cm}$ from the burst site; 2) the absorbing matter is located in the line of sight between the observer and the burster.

GRB 990705 has a duration of $\sim 42 \mathrm{~s}$ in the Gamma-Ray Burst Monitor (GRBM) and fluence $(9.3 \pm 0.2) \times 10^{-5} \mathrm{erg} \mathrm{cm}^{-2}$ in the 2-700 keV band (A2000). During the prompt phase, it shows an absorption feature at $3.8 \mathrm{keV}$ and an equivalent hydrogen column density, which disappears $13 \mathrm{~s}$ after the burst onset (A2000). This absorption feature was explained by A2000 as being due to an edge produced by neutral iron redshifted to $3.8 \pm 0.3 \mathrm{keV}$; the corresponding redshift is $0.86 \pm$ 0.17 . Optical spectroscopy of the host galaxy gives a redshift $z=0.8435$ (Andersen et al. 2002), consistent with the inferred value from the X-ray feature. This straightforward interpretation was, however, questioned by Lazzati et al. (2001) 
as it requires a vast amount of iron ${ }^{1}$ in the close vicinity of the burster. Lazzati et al. (2001) further suggested an alternative scenario in which the feature is produced by resonant scattering from hydrogen-like iron broadened by a range of outflow velocities. In this scenario, the radius of the $\mathrm{SN}$ shell is fixed by the requirement that the heating timescale of the electrons in the absorbing matter is $\sim 10 \mathrm{~s}$, i.e. $R_{\mathrm{s}} \sim(2-3) \times 10^{16} \mathrm{~cm}$. Our following work is based on this scenario.

A fading X-ray afterglow of GRB 990705 was detected by the Narrow Field Instruments of BeppoSAX 11 hours after the trigger, but the statistics are not sufficient to draw a detailed conclusion on the decaying law (A2000). Masetti et al. (2000) report having detected the counterpart of this burst twice in the near-infrared $H$ band and only once in the optical $V$ band, from a few hours to $\sim 1$ day after the GRB trigger. The first two $H$-band measurements define a power-law decay with in$\operatorname{dex} \alpha=1.68 \pm 0.10\left(F \propto t^{-\alpha}\right)$, but a third attempt to detect the source gave an upper limit, implying a much faster decay. No radio afterglow was detected (Subrahmanyan et al. 1999; Hurley et al. 1999).

For the afterglows with X-ray emission lines, the lineemitting gas could lie outside of the line of sight of the burst and therefore has no direct relation with the afterglow radiation. However, for afterglow with X-ray absorption features, the absorbing matter ( $\mathrm{SN}$ shell) should have a direct effect on the afterglow radiation, because it must lie in the line of sight of the burst. So, an examination of the self-consistency between the power-law afterglow and the X-ray absorption feature is quite necessary.

\section{Afterglow models for GRB 990705}

We here investigate the afterglow behavior of GRBs assuming the supranova-like scenario (Vietri \& stella 1998; Wang et al. 2000a) where a thick torus of matter (i.e. the supernova remnant shell) lies, in the line of sight of the burst, at a radius $R_{\mathrm{S}}$ from the burst center with a width $\Delta R_{\mathrm{s}}$ and particle density $n_{\mathrm{s}}$. We attempt to fit the $H$-band afterglow of GRB 990705, as a representative case. For uniform circum-burst medium, the deceleration radius, at which the energy of the hot, swept-up external medium by the blast wave equals that in the original explosion, of the GRB relativistic shell is (e.g. Piran 1999)

$R_{\mathrm{d}}=\left(\frac{3 E}{4 \pi \eta^{2} n m_{\mathrm{p}} c^{2}}\right)^{1 / 3}=6 \times 10^{16} \mathrm{~cm} E_{53}^{1 / 3} n_{0}^{1 / 3} \eta_{300}^{-2 / 3}$

where $E=10^{53} E_{53} \mathrm{erg}$ is the shell isotropic kinetic energy, $n=10^{0} \mathrm{~cm}^{-3}$ is the particle density of the circum-burst medium, and $\eta=300 \eta_{300}$ is the initial Lorentz factor of the shell. According to whether $R_{\mathrm{d}} \gg R_{\mathrm{S}}$ or $R_{\mathrm{d}} \ll R_{\mathrm{S}}$, there are two possible locations of the afterglow-emitting regions: one is in the torus on which the fireball impacts (case I) and the other is in the circum-burst medium inside the torus (case II).

${ }^{1}$ The required total mass of iron is $35 f M_{\odot}$ (see Eq. (5) in Lazzati et al. 2001), where $f$ is the covering factor of the absorbing material surrounding the burst.

\subsection{Case I: Jet-torus interaction model}

We assume that the torus has a width $\Delta R_{\mathrm{s}}$, density $n_{\mathrm{s}}=$ $M / 4 \pi R^{2} m_{\mathrm{p}}$ and scattering optical depth $\tau_{\mathrm{T}}=\sigma_{\mathrm{T}} n \Delta R_{\mathrm{s}} . \tau_{\mathrm{T}} \lesssim$ 1 must be satisfied to maintain the flickering behavior of the burst. Values consistent with this could be a few solar masses located at $R_{\mathrm{s}} \sim(2-3) \times 10^{16} \mathrm{~cm}$, which gives $\tau_{\mathrm{T}}=$ $0.67\left(M / 10 M_{\odot}\right)\left(R_{\mathrm{S}} / 3 \times 10^{16} \mathrm{~cm}\right)^{-2}$ and a particle density $n_{\mathrm{s}}=10^{9}\left(M / 10 M_{\odot}\right)\left(R_{\mathrm{s}} / 3 \times 10^{16} \mathrm{~cm}\right)^{-2}\left(\Delta R_{\mathrm{s}} / 10^{15} \mathrm{~cm}\right)^{-1}$.

The torus will be hit by the the fireball shell a few seconds $\left(\delta t \sim R_{\mathrm{s}} / 2 \eta^{2} c=2 s R_{\mathrm{s}, 16} \eta_{300}^{-2}\right.$, where $\left.R_{\mathrm{s}}=10^{16} R_{\mathrm{s}, 16} \mathrm{~cm}\right)$ after it is reached by the burst proper. The impact process has been described in Vietri et al. (1999), where the authors attempt to interpret the anomalous X-ray afterglow of GRB 970508 and GRB 970828. The impact of the fireball on the torus will generate a forward shock propagating into the torus, and a reverse one moving into the fireball shell. They predicted, during the impacting, a secondary burst from the reverse shock and a very short-lived forward shock for GRB 970508. However, we will show below that for GRB 990705, which has a much larger shock energy $E$, the forward shock could last few days (especially for the lower estimated value for the torus temperature given by Paerels et al. 2000, see Eq. (7)), giving rise to an early power-law fading afterglow as seen in GRB 990705. The disappearance of this forward shock may just account for the observed faster decline at the late time.

When the rest-mass-energy of the swept-up material equals the shock energy, the forward shock will be slowed down to non-relativistic speeds; this occurs after the shock has propagated a quite short distance $d$ in the torus ${ }^{2}$, where

$d=\frac{E}{4 \pi R_{\mathrm{s}}^{2} n_{\mathrm{s}} m_{\mathrm{p}} c^{2}}=5 \times 10^{12} \mathrm{~cm} E_{53} n_{\mathrm{s}, 10}^{-1} R_{\mathrm{s}, 16}^{-2}$,

and the corresponding time is

$t_{\mathrm{nr}}=\frac{d}{c}=160 \mathrm{~s} E_{53} n_{\mathrm{s}, 10}^{-1} R_{\mathrm{s}, 16}^{-2}$.

For an adiabatic shock, the conservation of energy is as follows

$E=4 \pi R_{\mathrm{s}}^{2} x n_{\mathrm{s}} m_{\mathrm{p}} v^{2} / 2=$ constant

where $x$ is the distance that the forward shock have propagated in the torus and $v$ is the shock velocity. From this equation and $t \sim x / v$, we get the scaling laws of the dynamic quantities: $v=c(x / d)^{-1 / 2}, v=c\left(t / t_{n r}\right)^{-1 / 3}$ and $x=d\left(t / t_{n r}\right)^{2 / 3}$. Please note that these dynamic relations are different from the usual Sedovvon Neumann-Taylor solution of a non-relativistic GRB shock (Wijers et al. 1997; Dai \& Lu 1999; Wang et al. 2000b) because here the fireball is decelerated in a dense shell with an almost fixed radius $R_{\mathrm{s}}$.

As the fireball slows down, the ram pressure of the shell $\left(P=\rho_{\mathrm{b}} v^{2}\right.$ where $\rho_{\mathrm{b}}=E / \eta c^{2} 4 \pi R_{\mathrm{s}}^{2} m_{\mathrm{p}} x_{\mathrm{b}}$ is the shell density) on the external torus matter decreases with time. The material in the torus is supposed to be brought up to a temperature $T_{\mathrm{s}} \sim 10^{7}-10^{8} \mathrm{~K}$ by heating/cooling from the proper burst and its afterglow radiation (Vietri et al. 1999;

\footnotetext{
2 The denominator of the formula of $d$ in Vietri et al. (1999) has an extra " $\eta$ ", so they got a much lower value for $d$.
} 
Paerels et al. 2000). Thus, at a certain distance $x_{\mathrm{b}}$, the strong counter-pressure $\left(\sim n_{\mathrm{s}} k T_{\mathrm{s}}\right)$ in the pre-shock torus equals the ram pressure and begins to damp down the forward shock. We expect that the forward shock emission decays exponentially with time since then. Equating $\rho_{\mathrm{b}} v^{2}$ with $n_{\mathrm{s}} k T_{\mathrm{s}}$ gives

$$
\begin{aligned}
x_{\mathrm{b}} & \simeq\left(\frac{E d}{8 \pi \eta R_{\mathrm{s}}^{2} n_{\mathrm{s}} k T_{\mathrm{s}}}\right)^{1 / 2} \\
& =2 \times 10^{14} \mathrm{~cm} E_{53} R_{\mathrm{s}, 16}^{-2} \eta_{300}^{-1 / 2} n_{\mathrm{s}, 10}^{-1} T_{\mathrm{s}, 7}^{-1 / 2} .
\end{aligned}
$$

The shock velocity at $x_{\mathrm{b}}$ is

$v_{\mathrm{b}}=c\left(x_{\mathrm{b}} / d\right)^{1 / 2}=0.16 c \eta_{300}^{1 / 4} T_{\mathrm{s}, 7}^{1 / 4}$.

So, the characteristic time when the forward shock vanishes is

$t_{\mathrm{b}} \sim x_{\mathrm{b}} / v_{\mathrm{b}}=4 \times 10^{4} \mathrm{~s} E_{53} R_{\mathrm{s}, 16}^{-2} \eta_{300}^{-3 / 4} n_{\mathrm{s}, 10}^{-1} T_{\mathrm{s}, 7}^{-3 / 4}$

after the burst (note that here $\delta t$ and $t_{\mathrm{nr}}$, compared to $t_{\mathrm{b}}$, are both negligible).

Up to now, we have assumed that the radial time scale of the fireball shell is relevant to the dynamic time scale. This requires that the angular spreading timescale does not dominate the radial time scale, i.e. $R_{\mathrm{s}} \theta_{j}^{2} / 2 c \lesssim x / v$, where $\theta_{j}$ is the opening angle of the fireball shell, which means that actually the outflow is a jet. The first measurement of the $H$-band afterglow is at $\sim 4$ hours after the burst, so $\theta_{j} \lesssim 0.3 R_{\mathrm{s}, 16}^{-1 / 2}$. Actually, a mildly collimated outflow is quite plausible considering the large isotropic gamma-ray energy of this burst. Please note that, in the jet-torus interaction model, sideways expansion of the jet in the torus cannot change the opening angle significantly as the sideways expansion length is much smaller than the radius $R_{\mathrm{s}}$, i.e. $\theta_{j}=\theta_{0}+c_{\mathrm{s}} t /\left(R_{\mathrm{s}}+v t\right) \simeq \theta_{0}$, where $c_{\mathrm{s}}$ is the sound velocity in the torus.

Now we investigate the fading behavior of the afterglow as the non-relativistic forward shock slows down in the torus. During this phase, the typical electron Lorentz factor is

$$
\begin{aligned}
\gamma_{m} & =\epsilon_{\mathrm{e}} \frac{(p-2)}{(p-1)} \frac{m_{\mathrm{p}}}{m_{\mathrm{e}}} \frac{v^{2}}{2 c^{2}} \\
& =60 \frac{(p-2)}{(p-1)} \epsilon_{e, 0.5} E_{53}^{2 / 3} n_{\mathrm{s}, 10}^{-2 / 3} R_{\mathrm{s}, 10}^{-4 / 3} t_{1 \mathrm{~h}}^{-2 / 3},
\end{aligned}
$$

where $\epsilon_{\mathrm{e}} \equiv 0.5 \epsilon_{\mathrm{e}, 0.5}$ is the fraction of the shock energy carried by the electrons and $t_{1 \mathrm{~h}}$ is the observing time in units of one hour. The post-shock magnetic field strength is

$$
\begin{aligned}
B & =\sqrt{8 \pi \epsilon_{B}\left(4 n_{\mathrm{s}} m_{\mathrm{p}} v^{2} / 2\right)} \\
& =100 \mathrm{G} \epsilon_{B,-4}^{1 / 2} n_{\mathrm{s}, 10}^{1 / 6} E_{53}^{1 / 3} R_{\mathrm{s}, 16}^{-2 / 3} t_{1 \mathrm{~h}}^{-1 / 3},
\end{aligned}
$$

where $\epsilon_{B} \equiv 10^{-4} \epsilon_{B,-4}$ is the fraction of the shock energy carried by the magnetic field. Thus we obtain the synchrotron peak frequency

$$
\begin{aligned}
v_{m} & =\frac{\gamma_{m}^{2} q_{\mathrm{e}} B}{2 \pi m_{\mathrm{e}} c} \\
& =10^{12} \mathrm{~Hz}\left(\frac{p-2}{p-1}\right)^{2} \epsilon_{e, 0.5}^{2} \epsilon_{B,-4}^{1 / 2} E_{53}^{5 / 3} n_{\mathrm{s}, 10}^{-7 / 6} R_{\mathrm{s}, 16}^{-10 / 3} t_{1 \mathrm{~h}}^{-5 / 3}
\end{aligned}
$$

where $q_{\mathrm{e}}$ is the electron charge, and the cooling frequency

$v_{\mathrm{c}}=6 \times 10^{10} \mathrm{~Hz} \epsilon_{B,-4}^{-3 / 2} n_{\mathrm{s}, 10}^{-1 / 2} E_{53}^{-1} R_{\mathrm{s}, 16}^{2} t_{1 \mathrm{~h}}^{-1}$.
The peak flux is

$F_{v_{m}}=\frac{1}{4 \pi d_{\mathrm{L}}^{2}} \frac{q_{\mathrm{e}}^{3}}{m_{\mathrm{e}} c^{2}} N_{\mathrm{e}} B \propto t^{1 / 3}$,

where $N_{\mathrm{e}}=4 \pi R_{\mathrm{s}}^{2} x n_{\mathrm{s}} \theta_{j}^{2} / 2$ is the total number of electrons swept-up by the forward shock, $\theta_{j}$ is the opening angle of the jet and $d_{\mathrm{L}}$ is the luminosity distance of the burst. According to these relations, we further derive the spectrum and the light curve:

$F_{v}=\left\{\begin{array}{ll}\left(v / v_{m}\right)^{-(p-1) / 2} F_{v_{m}} & \text { if } v_{\mathrm{c}}>v>v_{m} \\ \propto v^{-(p-1) / 2} t^{-5 p / 6+7 / 6} & \\ \left(v_{\mathrm{c}} / v_{m}\right)^{-(p-1) / 2}\left(v / v_{\mathrm{c}}\right)^{-p / 2} F_{v_{m}} & \text { if } v>v_{\mathrm{c}}>v_{m} \\ \propto v^{-p / 2} t^{-5 p / 6+2 / 3} & \end{array}\right.$.

Thus the $H$-band decay index of GRB 990705 before 1 day can be reproduced if $p \simeq 2.8$ and $v_{H}>v_{\mathrm{c}}>v_{m}$. In Fig. 1, we give an analytic fitting to the $H$-band afterglow. As discussed in Sect. 1, the radius of the torus is almost fixed by the requirement that the heating timescale of the electrons in the torus is $\sim 10 \mathrm{~s}$ and we set $R_{\mathrm{s}}=3 \times 10^{16} \mathrm{~cm}$. For a SN shell of $10 M_{\odot}$ mass with a typical width $\Delta R=10^{15} \mathrm{~cm}$ at this radius, its particle density is $n_{\mathrm{s}}=10^{9} \mathrm{~cm}^{-3}$. We also chose the shock energy to be $E=5 \times 10^{53} \mathrm{erg}$, according to the isotropic gamma-ray energy of GRB 990705 and a reasonable gamma-ray production efficiency of GRBs. The free parameters in this fitting are the two unknown energy equipartition factors $\epsilon_{\mathrm{e}}$ and $\epsilon_{B}$, and the opening angle of the jet $\theta_{j}$. We find that the following values for these parameters are consistent with the observations: $\epsilon_{\mathrm{e}}=0.5$, $\epsilon_{B}=10^{-5}$ and $\theta_{j}=0.2$. The solid line in Fig. 1 represents the power-law decay of the afterglow as the forward shock slows down in the torus and the thick dotted line represents the later exponential decay $\left(F_{v} \propto \exp (-t)\right)$ of this shock due to the counter-pressure in the hot torus. With these parameters, the synchrotron self-absorption frequency scales with time as

$v_{a}=930 \mathrm{GHz}(t / 1 d)^{-(6-5 p) / 3(p+4)}$.

Such a large synchrotron self-absorption frequency is consistent with the non-detection of the radio afterglow.

The bremsstrahlung cooling time of the torus of density $n \sim 10^{9} \mathrm{~cm}^{-3}$ is given by

$t_{\mathrm{br}}=7 \times 10^{5} \mathrm{~s}_{9}^{-1} T_{\mathrm{s}, 7}^{1 / 2}$,

so the hot torus does not cool significantly during the phase of the interaction between the jet and the torus.

\subsection{Case II: Jet in a dense circum-burst medium}

The steepness of the light curve decay could also be produced by a beamed outflow (e.g. Rhoads 1999; Sari et al. 1999). The beam reduces the energy budget, alleviating the "energy crisis" of GRBs. Assuming that a break due to jet sideways spreading occurs in the $H$-band light curve of GRB 990705 about one day after the burst, the early time slope $\alpha \simeq 1.68$ and the later one $\alpha^{\prime}>2.6$ (based on the second $H$-band detection and the third $H$-band upper limit) would be consistent with $p \sim 2.9$. The thin dotted line in Fig. 1 represents this later power-law 
Table 1. The spectra and light curves for the different scenarios discussed in the text. $t_{\mathrm{b}}$ is the light curve break time of the afterglows. The parameter free relation between the spectral index and the light curve index can be derived by eliminating $p$ for each case.

\begin{tabular}{|c|c|c|c|}
\hline & \multirow{2}{*}{ Case I } & \multicolumn{2}{|c|}{ Case II } \\
\hline & & jet & relativistic to non-relativistic \\
\hline$t<t_{\mathrm{b}}, v<v_{\mathrm{c}}$ & $v^{-(p-1) / 2} t^{-(5 p-7) / 6}$ & $v^{-(p-1) / 2} t^{-3(p-1) / 4}$ & $v^{-(p-1) / 2} t^{-3(p-1) / 4}$ \\
\hline$t<t_{\mathrm{b}}, v>v_{\mathrm{c}}$ & $v^{-p / 2} t^{-(5 p-4) / 6}$ & $v^{-p / 2} t^{-(3 p-2) / 4}$ & $v^{-p / 2} t^{-(3 p-2)^{\prime} 4}$ \\
\hline$t>t_{\mathrm{b}}, v<v_{\mathrm{c}}$ & $v^{-(p-1) / 2} \exp (-t)$ & $v^{-(p-1) / 2} t^{-p}$ & $v^{-(p-1) / 2} t^{(21-15 p) / 10}$ \\
\hline$t>t_{\mathrm{b}}, v>v_{\mathrm{c}}$ & $v^{-p / 2} \exp (-t)$ & $v^{-p / 2} t^{-p}$ & $v^{-p / 2} t^{(4-3 p) / 2}$ \\
\hline
\end{tabular}

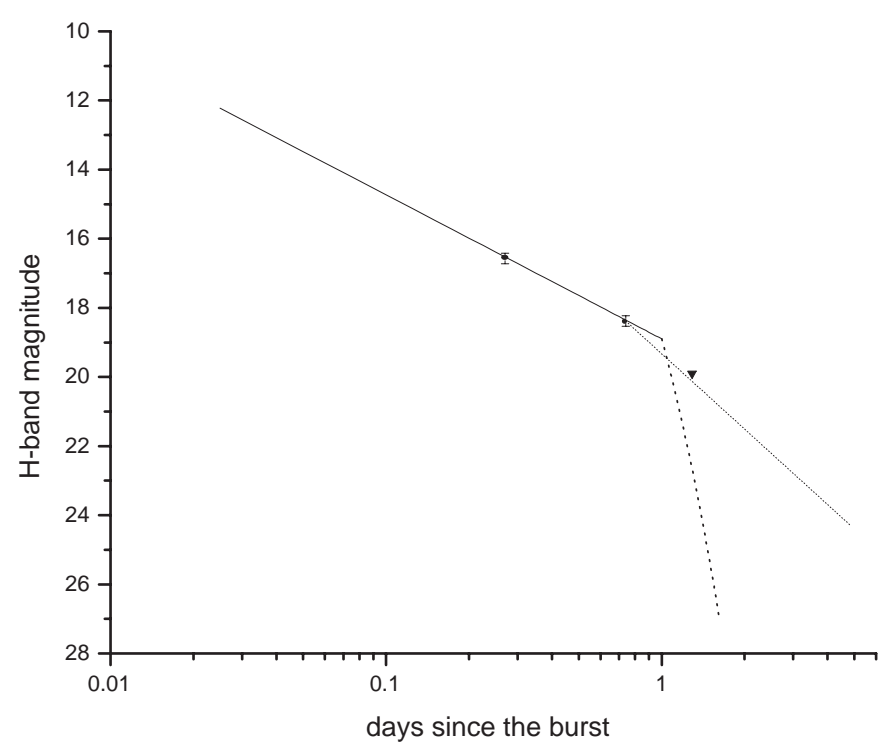

Fig. 1. An analytic fitting of the $H$-band afterglow of GRB 990705 in terms of the jet-torus interaction model (see text for details). Detections, and upper limits for the non-detections, taken from Masetti et al. (2000), are indicated by the filled circles with error bars $(3 \sigma)$ and arrows, respectively. The solid line represents the power-law decay of the afterglow as the forward shock slows down in the torus, and the thick dotted line represents the late exponential decay of this shock due to the effect of the counter-pressure in the hot torus. The thin dotted line represents the late power-law decay $F_{v} \propto t^{-p}$ due to jet behavior. See the text for the parameters used in this fitting.

decay $F_{v} \propto t^{-p}$ due to jet sideways expansion behavior. The sideways expansion of the jet makes its bulk Lorentz factor $\Gamma$ slow down exponentially with radius after a characteristic value $\theta_{j}^{-1}$. Afterwards, $\Gamma \propto \exp \left(-r / R_{\mathrm{b}}\right)$, where $R_{\mathrm{b}}$ is the shock radius at the time $\Gamma=\theta_{j}^{-1}$. For a uniform circum-burst medium, we have $\Gamma=\left(17 E / 1024 \pi n m_{\mathrm{p}} c^{5} t^{3}\right)^{1 / 8}$ (Sari et al. 1998), and

$R_{\mathrm{b}}=\left(\frac{17 E_{0}}{8 \pi n m_{\mathrm{p}} c^{2}}\right)^{1 / 3}=7 \times 10^{17} \mathrm{~cm} E_{0,51}^{1 / 3} n_{0}^{-1 / 3}$

where $E_{0}$ is the actual energy of the jet, $E_{0}=E_{\text {iso }} \theta_{j}^{2} / 2$. If the early power-law decaying afterglow is assumed to be produced by the deceleration of the jet in the circum-burst medium before it hits the surrounding torus, we require $R_{\mathrm{b}}<R_{\mathrm{S}} \sim 3 \times 10^{16} \mathrm{~cm}$. This means that the circum-burst medium has a number density $n \gtrsim 10^{4}-10^{5} \mathrm{~cm}^{-3}$, even if the actual energy of the burst is only
$E_{0} \sim$ a few $\times 10^{51} \mathrm{erg}$ as found by Frail et al. (2001). Frail et al. (2001) have inferred the jet opening angle $\theta_{j} \simeq 0.054$ from the light curve break time, assuming an interstellar medium of density $n=0.1 \mathrm{~cm}^{-3}$. A much larger circum-burst medium density leads to an energy reservoir an order of magnitude larger than what estimated by Frail et al. (2001), as $E_{0} \propto \theta_{j}^{2} \propto n^{1 / 4}$. A much larger density than that of a typical interstellar medium is also suggested by Ghisellini et al. (2002) from the point of view of constraining the total energy reservoir of GRB 991216 with emission line luminosity.

Afterglow light curve breaks can also be produced by spherical fireball expansion which undergoes a transition from a relativistic phase to a non-relativistic one (Wijers et al. 1997; Dai \& Lu 1999; Livio \& Waxman 2000). The power-law decay indices before and after the break are consistent with $p \sim 3.2$ if the $H$-band frequency is located between the characteristic break frequency and the cooling break frequency during the first day after the burst (see Eqs. (5) and (6) of Dai \& Lu 1999). This scenario also requires that at least the Sedov length of the shock $R_{\mathrm{nr}}$ is less than the torus radius. As

$R_{\mathrm{nr}}=\left(\frac{E_{\mathrm{iso}}}{4 \pi / 3 n m_{\mathrm{p}} c^{2}}\right)^{1 / 3}=2.5 \times 10^{18} \mathrm{~cm} E_{\mathrm{iso}, 53}^{1 / 3} n_{0}^{-1 / 3}$

where $E_{\text {iso }}$ is the isotropic kinetic energy of burst, this run means that $n \gtrsim 10^{6} \mathrm{~cm}^{-3}$. Such a large number density $(n \gtrsim$ $10^{4}-10^{6} \mathrm{~cm}^{-3}$ ) is typical of molecular clouds in star forming regions, independently supporting that long GRBs are connected with massive progenitors.

Due to the lack of detection after about one day for GRB 990705, we do not know the later behavior of its afterglow, hence we could not tell these scenarios from each other for this burst. However, we predict different types of behavior of afterglows for the different geometries discussed above. If we could know the spectra and light curves of the afterglows (for those GRBs that have absorption features) both before and after the break in future better observations, we can then have a more definite conclusion. The difference in the spectrum and light curve for these different scenarios are summarized in Table 1 .

\section{Conclusions and discussions}

Emission or absorption features in the X-ray spectrum of GRBs and their afterglows provide a useful tool for studying the 
close environment of GRBs and thus their possible progenitors. The absorption feature in the prompt X-ray emission of GRB 990705 was originally interpreted by Amati et al. (2000) to be a photoionization $\mathrm{K}$ edge of neutral iron. However, this straightforward explanation is shown by Lazzati et al. (2001) to require an improbably large amount of iron in the close environment of the burster. Instead, Lazzati et al. (2001) interpret this as a resonant absorption line broadened by a large spread of velocities. In this scenario, the disappearance of the feature $13 \mathrm{~s}$ after the burst results from electron heating due to the illuminating photons and it severely constrains the radius of the absorbing materials $\left(R \sim 2-3 \times 10^{16} \mathrm{~cm}\right.$, see Eq. (13) of Lazzati et al. 2001). A reasonable scenario for this requirement is the supranova-like scenarios (Vietri \& Stella 1998; Wang et al. 2000a), in which a young supernova remanent is located at the close vicinity of the burster. Based on these studies, in this paper we investigated whether the circum-burst environment constrained by the absorption feature could be consistent with the observed afterglows of GRB 990705.

We discussed two possible locations of the afterglowemitting region: one is in the torus where the afterglows are produced by the impact of the fireball jet on this torus and the other is in the dense circum-burst medium inside the torus. In the former scenario, the impact of the fireball on the torus will generate a forward shock propagating into the torus. This forward shock will be decelerated by the dense matter in the torus into a sub-relativistic phase in quite a short time and to a lower and lower velocity as time elapses. The heating/cooling processes of the torus by the burst and afterglow photons may bring its temperature to $T_{\mathrm{s}} \sim 10^{7} \mathrm{~K}$. Once the ram pressure $\left(\sim \rho_{\mathrm{b}} v^{2}\right)$ of the fireball falls low enough to be equal to the thermal counter-pressure $\left(n_{\mathrm{s}} k T_{\mathrm{s}}\right)$ of the hot torus, the forward shock is damped down very rapidly (Vietri et al. 1999) and the afterglow emission will cut off accordingly. We found that the $H$-band afterglow of GRB 990705 can be fitted in terms of this model.

In the latter scenario, as in many other afterglows, the steepening of the light curve decay of GRB 990705 one day after the burst is attributed to jet evolution in a uniform density medium or a spherical fireball undergoing a transition to nonrelativistic expansion. The broken power-law decay behavior of the $H$-band afterglow requires the shock radius at the light curve break time or at the Sedov phase, respectively, to be smaller than the torus location. This in turn requires that the circum-burst medium density must be $n \gtrsim 10^{4}-10^{5} \mathrm{~cm}^{-3}$ or $n \gtrsim 10^{6} \mathrm{~cm}^{-3}$, respectively. In this scenario, the fireball will also hit the surrounding torus finally. The abrupt density jump might cause a rise and a successive decline in the afterglows (see Dai \& Lu 2002 for a relativistic case).

A noticeable point relevant to the high density circum-burst medium is that the true energy reservoir of GRB 990705 may be much greater than what was estimated by Frail et al. (2001), $E_{\gamma}=3.9 \times 10^{50} \mathrm{erg}$, derived from the jet model by assuming an interstellar medium of density $n=0.1 \mathrm{~cm}^{-3}$, since the calculated fireball true energy depends on $\theta_{j}^{2}$ which in turn depends on $n^{1 / 4}$.
In summary, the geometry requirement of the X-ray absorption feature of GRB 990705 is shown to be also consistent with its afterglows, although the sparse data of the afterglow makes it impossible to reach a definite conclusion on the two scenarios. Future better broad-band observations of the afterglow spectra and light curves for GRBs that have absorption features could tell which one is true and thereby provides a more valuable insight into the environment and the central engine.

Acknowledgements. We are grateful to the referee for his constructive and careful comments. XYW would like to thank $\mathrm{Z}$. Li for valuable discussions. This work was supported by the Special Funds for Major State Basic Research Projects, the National Natural Science Foundation of China under grants 19973003 and 19825109, 10233010 and the National 973 project (NKBRSF G19990754).

\section{References}

Amati, L., Frontera, F., Vietri, M., et al. 2000, Science, 290, 953

Andersen, M. I., et al. 2002, in preparation

Antonelli, L. A., Piro, L., Vietri, M., et al. 2000, ApJ, 545, L39

Böttcher, M., \& Fryer, C. L. 2001, ApJ, 547, 338

Böttcher, M., Fryer, C. L., \& Dermer, C. D. 2002, ApJ, 567, 441

Dai, Z. G., \& Lu, T. 1999, ApJ, 519, L155

Dai, Z. G., \& Lu, T. 2002, ApJ, 565, L87

Frail, D. A., Waxman, E., \& Kulkarni, S. R. 2000, ApJ, 537, 191

Frail, D. A., Kulkarni, S. R., Sari, R., et al. 2001, ApJ, 562, L55

Ghisellini, G., Lazzati, D., Rossi, E., \& Rees, M. J. 2002, A\&A, 389, L33

Hurley, K., \& Feroci, M. 1999, GCN Circ., 378 http://gcn.gsfc.nasa.gov/gcn/gcn3/378.gcn3

Lazzati, D., Campana, S., \& Ghisellini, G. 1999, MNRAS, 304, L31

Lazzati, D., Ghisellini, G., Amati, L., et al. 2001, ApJ, 556, 471

Lazzati, D., Ramirez-Ruiz, E., \& Rees, M. J. 2002, ApJ, 572, L57

Livio, M., \& Waxman, E. 2000, ApJ, 538, 187

Masetti, N., Palazzi, E., Pian, E., et al. 2000, A\&A, 354, 473

Mészáros, P., \& Rees, M. J. 1998, MNRAS, 299, L10

Mészáros, P., \& Rees, M. J. 2001, ApJ, 556, L37

Paerels, F., Kuulkers, E., Heise, J., \& Liedahl, D. A. 2000, ApJ, 535, L25

Piran, T. 1999, Phys. Rep., 314, 575

Piro, L., Costa, E., Feroci, M., et al. 1999, ApJ, 514, L73

Piro, L., Garmire, G., Garcia, M., et al. 2000, Science, 290, 955

Piro, L. 2002 [astro-ph/0203275]

Rees, M. J., \& Mészáros, P. 2000, ApJ, 545, L73

Reeves, J., Watson, D., Osborne, J. P., et al. 2002, Nature, 416, 512

Rhoads, J. E. 1999, ApJ, 525, 737

Sari, R., Piran, T., \& Narayan, R. 1998, ApJ, 497, L17

Sari, R., Piran, T., \& Halpern, J. P. 1999, ApJ, 518, L17

Subrahmanyan, R., Cunningham, S. J., Wieringa, M. H., Frail, D. A., \& Kulkarni, S. R. 1999, GCN Circ., 376

http://gcn.gsfc.nasa.gov/gcn/gcn3/376.gcn3

Vietri, M., \& Stella, L. 1998, ApJ, 507, L45

Vietri, M., Perola, G. C., Piro, L., \& Stella, L. 1999, MNRAS, 308, L29

Vietri, M., Ghisellini, G., Lazzati, D., et al. 2001, ApJ, 550, L43

Wang, X. Y., Dai, Z. G., Lu, T., Wei, D. M., \& Huang, Y. F. 2000a, A\&A, 357, 543

Wang, X. Y., Dai, Z. G., \& Lu, T. 2000b, MNRAS, 317, 170

Weth, C., Mészáros, P., Kallman, I., \& Rees, M. J. 2000, ApJ, 534, 581

Wijers, R. A. M. J., Rees, M. J., \& Mészáros, P. 1997, MNRAS, 288, L51 